\title{
LA GESTIÓN INTEGRADA DE LOS RECURSOS HÍDRICOS EN LAS ISLAS BALEARES
}

\author{
Antoni Rodríguez Perea y Bernadí Gelabert Ferrer \\ Departament de Ciències de la Terra \\ Universitat de les Illes Balears
}

\section{RESUMEN}

En el Mediterráneo, los recursos hídricos dependen en gran medida de las precipitaciones y éstas varían tanto a largo del año, como en secuencias hiperanuales. La gestión hídrica ha de adaptarse a este carácter cíclico para ser sostenible y se deben gestionar los diversos recursos disponibles teniendo en cuenta dichas oscilaciones. Sólo utilizando la inercia y la capacidad de almacenamiento de los acuíferos, junto a recursos no convencionales, pueden afrontarse con éxito los inevitables periodos de sequía. En las Baleares, una gestión integral de todos los recursos disponibles tanto convencionales como no convencionales, apoyada en una buena gestión de la demanda, resultó fundamental para poder superar la falta de precipitaciones (hasta un 50\% por debajo de la media) del periodo 1998-2001 y se presenta como la mejor solución para la gestión de los recursos futuros.

Palabras clave: Recursos hídricos, Gestión integral, Islas Baleares, Recarga de acuíferos, Recursos hídricos no convencionales.

\begin{abstract}
In the Mediterranean, the water resources mainly depend on rainfall which presents both, annual and hyper annual variations. Sustainable water management needs to adapt to this cycle variations and to manage the available resources according to this rainfall cycles. Only the use of the inertia and the storage capacity of the aquifers, with non conventional resources, can successfully face the inevitable drought periods. In the Balearic Islands, an integrated management of all resources, conventional and non conventional, combined with a good water demand management, resulted fundamental in overcoming the lack of precipitation (50\% under the mean) during the 1998-2001 period and is presented as the best way to solve the future resources management.
\end{abstract}

Key words: Water resources, Integrated management, Balearic Islands, Aquifer recharge, Non conventional water resources. 


\section{Introducción}

Los ciclos climáticos en las regiones mediterráneas presentan oscilaciones anuales de temperatura y precipitación. Históricamente, esta característica ha obligado a una gestión anual de los recursos hídricos. Para ello, las aguas pluviales han sido tradicionalmente almacenadas en aljibes para el suministro domestico y en balsas para el riego agrícola, mientras la demanda urbana era abastecida a través de acueductos que conducían el agua desde manantiales próximos. Las zonas de agricultura intensiva se proveían mediante acequias desde las fuentes cercanas o desde pozos. No obstante, el incremento de la población y de su consumo hizo insuficiente el abastecimiento tradicional. El desarrollo tecnológico permitió la construcción de pantanos cada vez mayores y de pozos cada vez más profundos. La generalización del uso de bombas hidráulicas posibilita incrementar las extracciones de los acuíferos y favorece la ejecución de proyectos cada vez mayores que aumentan la explotación de recursos cada vez más lejanos. No obstante, todo ello se produce a costa de impactos ambientales cada vez más amplios y de la sobreexplotación de los recursos superficiales y subterráneos.

En este contexto, el modelo basado en la oferta ilimitada de agua entra en crisis, sus costes económicos y ambientales crecen de forma exponencial y hacen inviables los nuevos proyectos. Consecuencia de ello, se plantea la necesidad de gestionar la demanda de agua y de revisar la eficiencia de la gestión actual del agua. Una Nueva Cultura del Agua se abre paso en la política hidráulica del país rompiendo el monopolio tecnocrático existente y considerando el ciclo del agua, no de una forma aislada, sino dentro de un análisis ecointegral que considera el ciclo energético en toda su globalidad. La gestión integral de los recursos hídricos, incluyendo la gestión de la demanda y considerada dentro del ciclo planetario, se presenta como la alternativa más eficiente para superar la crisis existente.

\subsection{El carácter cíclico de los recursos hídricos}

Al enfrentarnos a los problemas específicos de las zonas mediterráneas, hemos de considerar, en primer lugar, los ciclos climáticos característicos de dichas áreas. Si observamos la gráfica de precipitaciones acumuladas en Mallorca (fig. 1), representativa de muchas de las zonas mediterráneas, resultan evidentes, no sólo las oscilaciones invierno-verano, sino

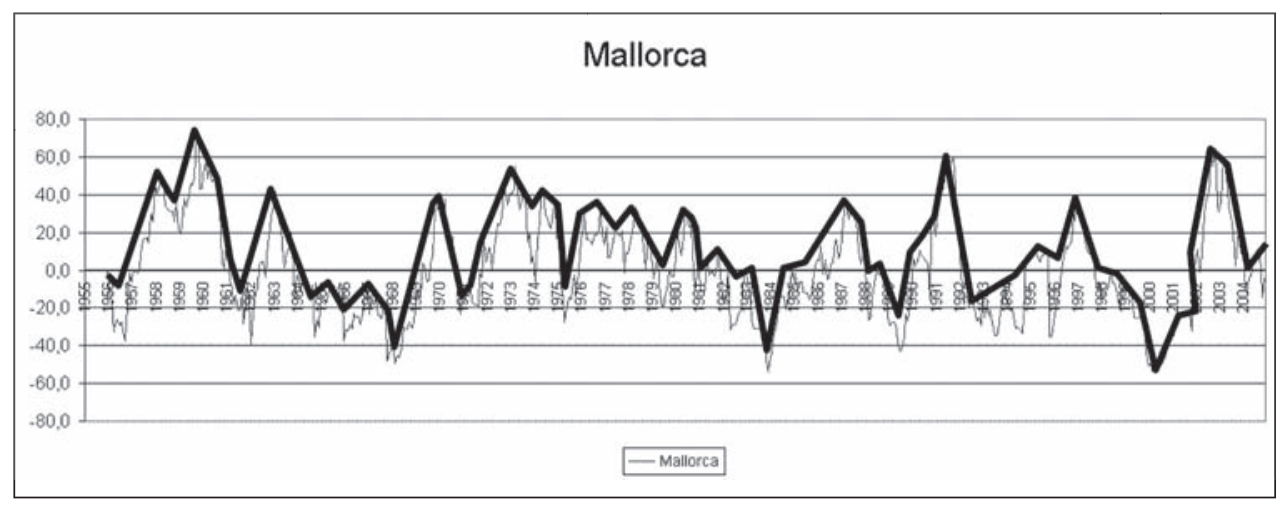

FIGURA 1: Precipitación anual acumulada en Mallorca en relación con el promedio, entre 1955 y 2005, según el Centro Territorial en Palma de Mallorca del INME. 
también oscilaciones hiperanuales, es decir, una alternancia entre ciclos secos, cuya duración media es, en nuestro caso, de 2,4 años, y ciclos húmedos con una duración promedio de 2 años.

\subsection{El desfase entre la oferta y la demanda}

Tanto en el consumo urbano, como en el agrícola, la demanda de agua presenta una curva que se sitúa en desfase con la precipitación, es decir, la máxima demanda anual se produce en verano y en los años secos. Sin embargo, muchas infraestructuras de abastecimiento, especialmente las que tienen como función preferente el almacenamiento, están dimensionadas para cubrir la máxima demanda anual, es decir para las puntas del verano.

Si tenemos en cuenta la ciclicidad de la demanda y la vista de la gráfica de precipitaciones, resulta evidente la necesidad de una gestión del almacenamiento hídrico hiperanual. Para ello, en numerosas regiones se ha usado y abusado de los pantanos, olvidando la capacidad de almacenamiento de los acuíferos. En otras, especialmente en el litoral, se intenta solucionar el problema entre oferta y demanda mediante la desalación. No es objeto de este artículo el análisis de las ventajas e inconvenientes de tales infraestructuras, no obstante, baste ahora señalar algunas de sus principales características.

Los embalses representan con toda seguridad una reserva hídrica de primera categoría en la península Ibérica, sin embargo, sus costes económicos y sociales han sido muchas veces infravalorados en comparación con sus beneficios. De hecho, las posibilidades de nuevos embalses cuya rentabilidad quede probada, más allá de dudas razonables, son extremadamente bajas en la Península y nulas en las Islas Baleares. La desalación, a pesar de los avances técnicos recientes, continua representando trasladar el problema del agua al consumo de energía. La gestión de la demanda, junto a una utilización eficiente de todos los recursos hídricos existentes son las únicas alternativas sostenibles a la situación crítica del agua en los países mediterráneos.

\subsection{Las aguas subterráneas}

A pesar que en zonas como las islas Baleares los recursos subterráneos proveen casi la totalidad de las necesidades hídricas, en otras regiones su utilización es muy limitada. Si consideramos su capacidad de almacenamiento la diferencia es abismal. La comparación entre el volumen de agua dulce almacenada en los acuíferos y la que se encuentra en forma liquida en la superficie del planeta (fig. 2) nos proporciona una buena imagen de la capacidad de almacenamiento de los acuíferos: las aguas superficiales representan el 0,3\% del agua dulce de la Tierra, mientras que las subterráneas llegan al 31,4\%, es decir, en un determinado momento existe 104 veces más aguas subterráneas que superficiales. A pesar de que el ciclo de renovación de las aguas subterráneas es mucho más lento que el de las superficiales, el volumen de las primeras es enorme comparado con las segundas.

No obstante, muchos acuíferos presentan problemas similares a los de las aguas superficiales: la contaminación de las aguas subterráneas es un problema creciente en las regiones mediterráneas, también lo es la sobreexplotación y en el caso de los acuíferos litorales, la intrusión salina representa una combinación de los dos problemas anteriores demasiado frecuente. A pesar de ello, su capacidad para almacenar recursos hídricos permanece casi intacta y prácticamente sin utilizar. Más aún, cuando la sobreexplotación a lo largo de décadas ha disminuido sus reservas de tal forma, que la infiltración del agua de lluvia no consigue su recuperación. Este sería el caso del acuífero de s'Estremera en Mallorca (fig. 3), cuya piezometría muestra una gráfica descendente, en 'diente de sierra', en la que cada 


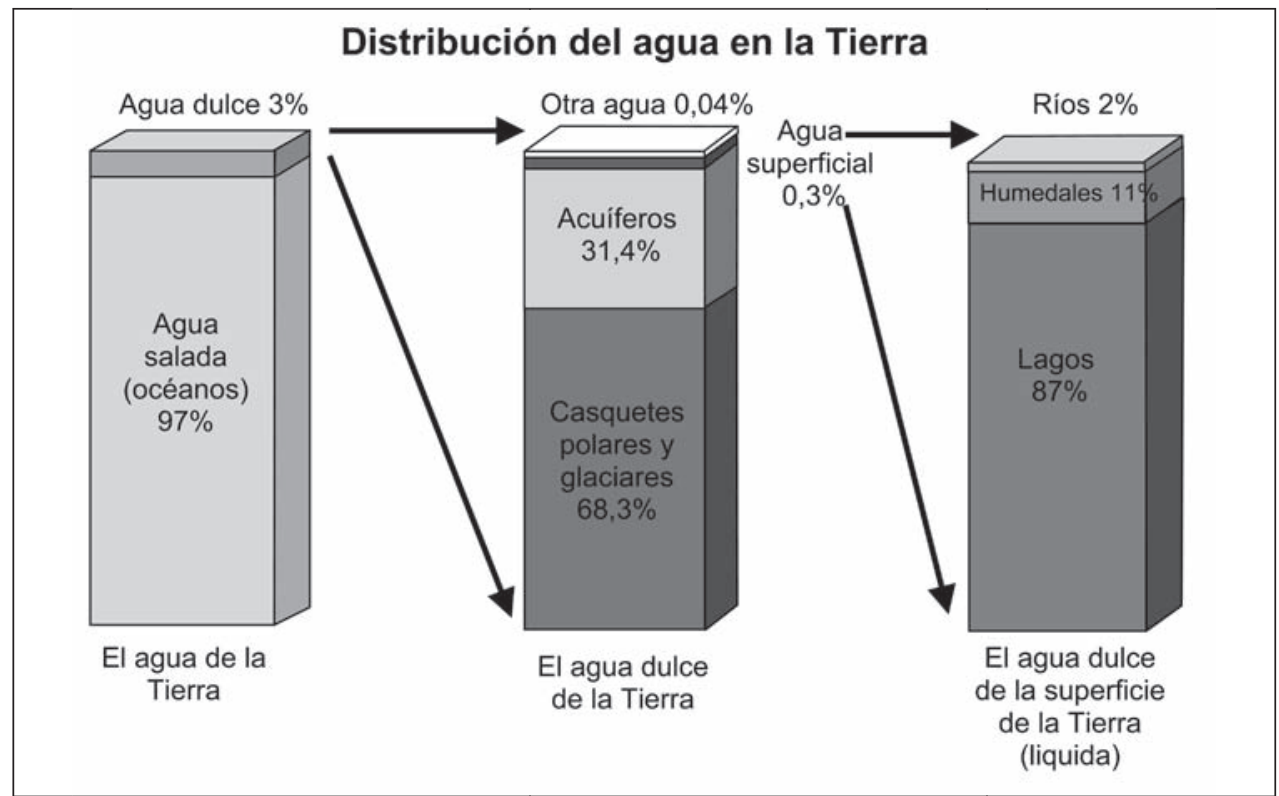

FIGURA 2: Distribución de los recursos hídricos en el planeta según GLEICK (1996) y el USGS (2004).

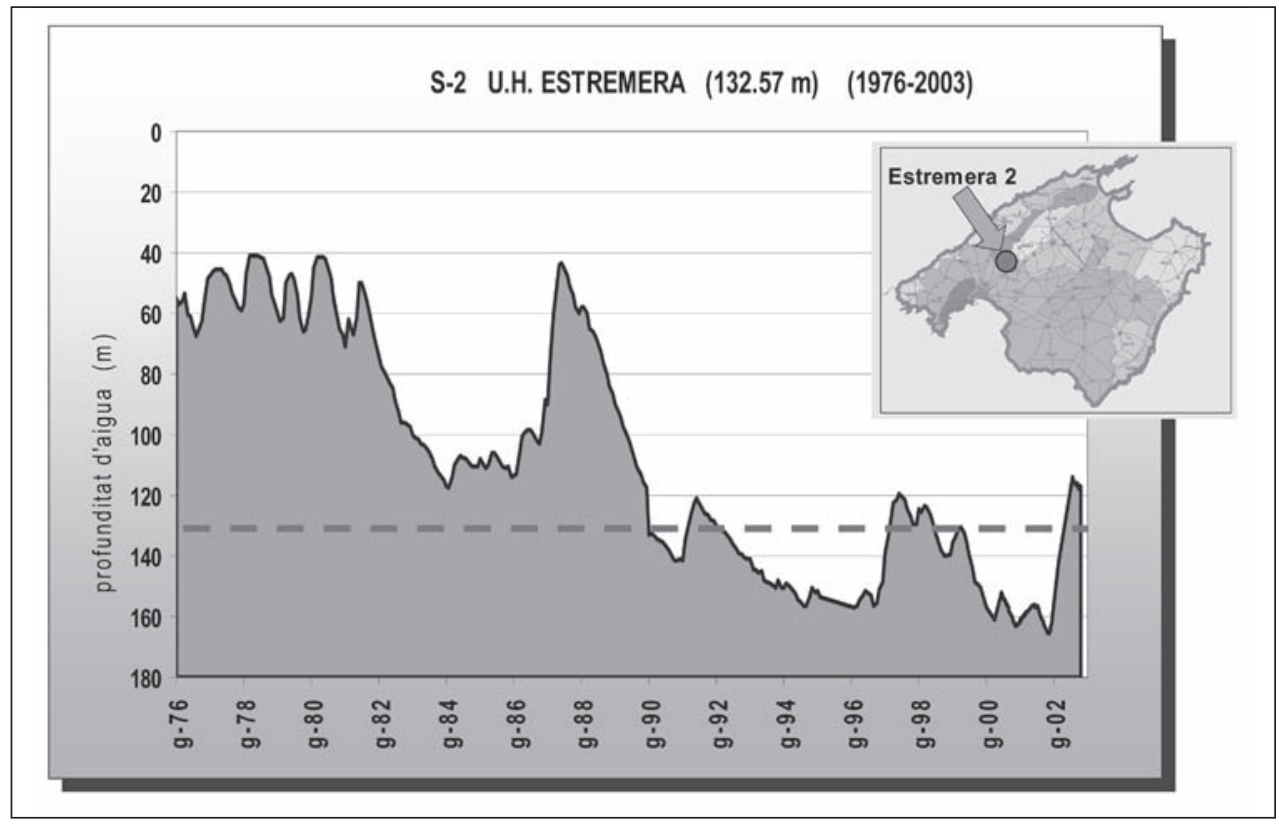

FIGURA 3: Evolución piezométrica representativa del acuífero de S'Estremera entre 1976 y 2002. La línea de trazos corresponde a la cota del nivel del mar. El ascenso que tiene lugar a partir del 2002 corresponde al inicio de un nuevo periodo lluvioso y sobre todo a la infiltración artificial de agua en el acuífero. 
episodio lluvioso no logra más que ralentizar el descenso del nivel hasta la próxima sequía. Por todo ello, en zonas de clima mediterráneo, una gestión integrada hiperanual ha de considerar la capacidad de almacenamiento de los acuíferos de forma prioritaria.

\subsection{Los recursos para una gestión integrada}

El principal objetivo de una gestión integrada es el considerar el uso de todos los recursos hídricos disponibles de la manera más eficiente. Ello requiere priorizar cada uno de ellos de acuerdo con sus características y sus potenciales, basándonos no solo en criterios económicos, sino también en criterios de sostenibilidad; esto es con el objetivo de recuperar los ecosistemas acuáticos ya que este último objetivo representa la mejor garantía de suficiencia y cualidad del recurso.

Entre otros deberemos contemplar como recursos: las aguas superficiales, las aguas pluviales, las aguas subterráneas, las aguas desalinizadas, la gestión de la demanda, las aguas depuradas, las aguas regeneradas, etc.

\section{La integración de las aguas desalinizadas}

La desalación de aguas salinas es un proceso relativamente moderno que ha cobrado actualidad al extenderse su utilización, especialmente en las zonas litorales y en los archipiélagos. Los avances técnicos han reducido drásticamente su consumo de energía pasando en pocos años de $6 \mathrm{kw} / \mathrm{m}^{3}$ a menos de $3 \mathrm{kw} / \mathrm{m}^{3}$. No obstante, en muchas ocasiones sus capacidades y su utilización se han orientado a cubrir el déficit del abastecimiento tradicional. Así, en las zonas turísticas, como las islas Baleares, se han diseñado plantas para abastecer la punta de demanda de núcleos turísticos, intentando evitar los efectos de las sequías sobre su abastecimiento. Con ello, estos recursos no convencionales se ven infrautilizados durante el resto del año y así, su potencial, como recuperador de los ecosistemas acuáticos sobrexplotados, ha sido dilapidado.

En la figura 4 se representa de forma esquemática la típica evolución de la demanda en una zona turística de clima mediterráneo. El ciclo anual presenta una punta estival y un valle invernal. En cambio, el ciclo hiperanual presenta, de forma muy poco previsible, una serie de años con precipitaciones por encima de la media y demanda baja, tanto en invierno como en verano, seguidos de otros años, caracterizados por lluvias por debajo de la media, y una alta demanda.

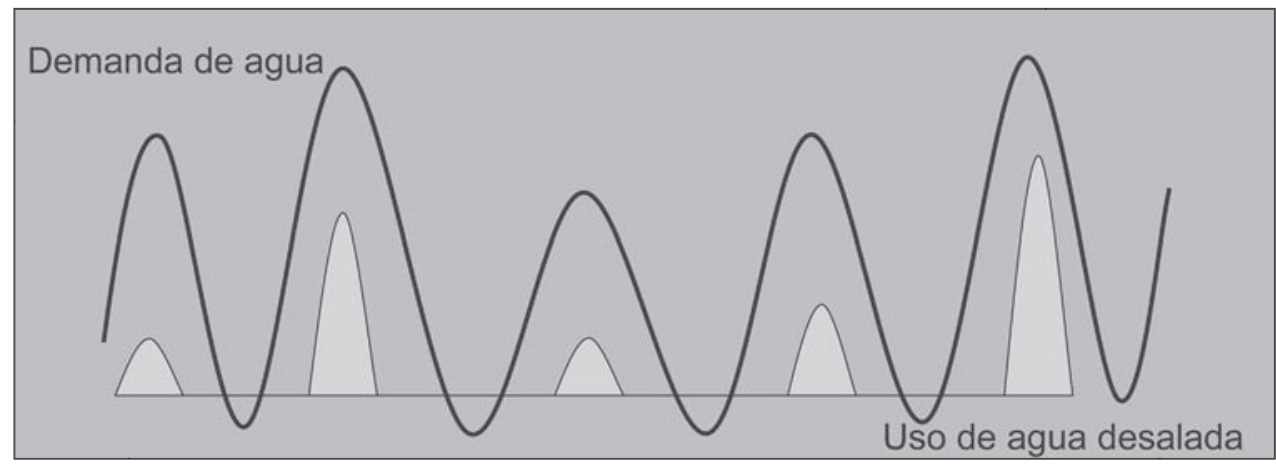

FIgURA 4: Esquema de la utilización del agua desalada en relación con la demanda total de agua. 
El consumo de agua desalada, en este modelo esquemático, sigue, en una gestión tradicional, el mismo carácter cíclico que la demanda, es decir, es prácticamente nulo en invierno y alcanza una mayor o menor producción en la punta de verano.

Un ejemplo de este tipo de gestión puede identificarse en la comparación de la producción mensual de la planta desaladora de agua salobre de son Tugores en Palma de Mallorca entre 1995 y 2004, con la grafica de precipitaciones en los mismos años (figura 5).

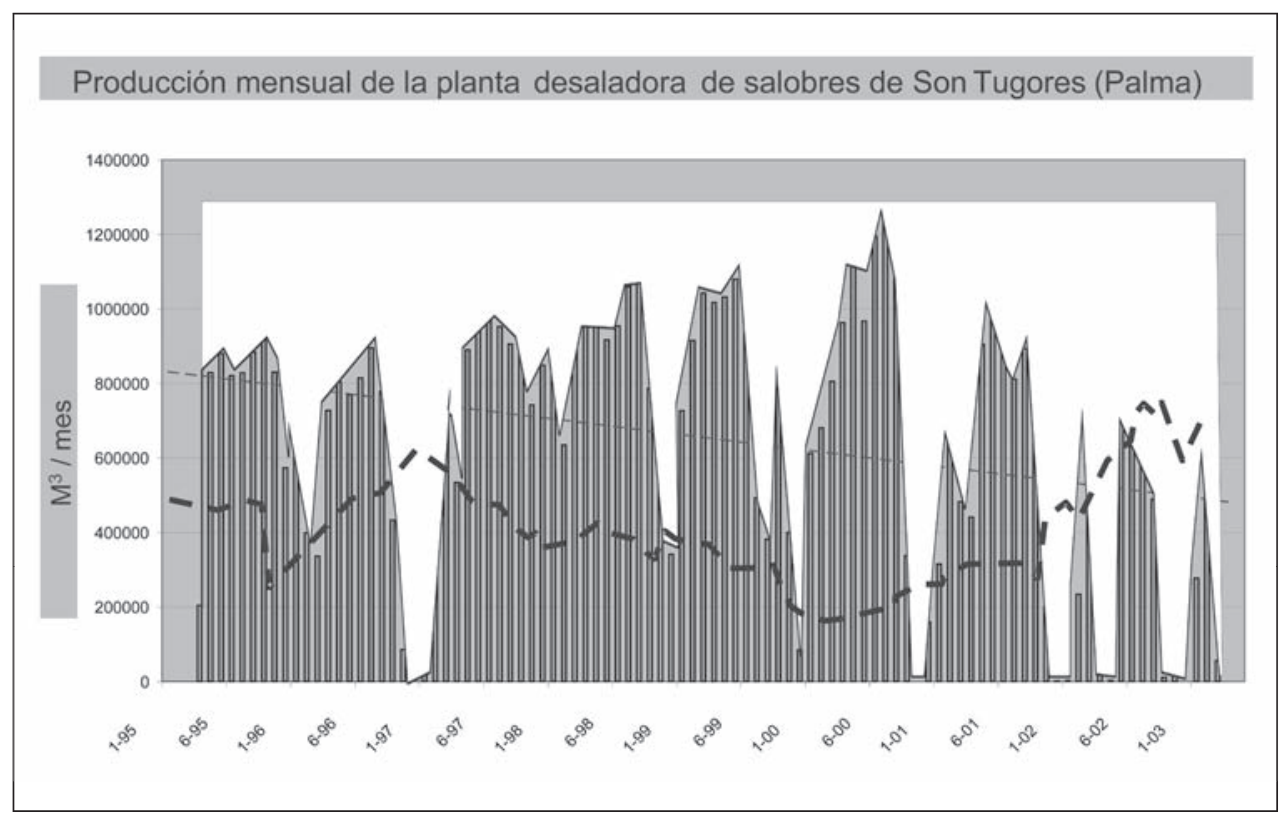

Figura 5: Comparación entre la producción mensual de la planta desaladora de son Tugores (Palma de Mallorca) y la gráfica de precipitación anual acumulada (línea de trazos) en idéntico periodo.

Los datos muestran puntas de producción creciente en los veranos del 1995, 1996, 1997, 1998, 1999 y 2000 y decreciente en los veranos de 2001, 2002 y 2003. Los primeros corresponden al avance de un periodo seco que se inicia en el 1997 y remite en el 2001. Los segundos se corresponden con el aumento de precipitaciones que se produce a partir de noviembre del 2001. La producción invernal es prácticamente cero en los años lluviosos: en 1997, el año en que se inicia el periodo seco y en 2001, 2002 y 2003, es decir, en los inviernos del siguiente periodo lluvioso que empezó en el 2001.

Tanto el modelo esquemático de la figura 4, como los datos reales de la producción de la desaladora de son Tugores (fig. 5), muestran que la gestión de las plantas desaladoras, en zonas mediterráneas con fuerte peso turístico, presentan excedentes de producción durante la temporada baja. Una simulación basada en la frecuencia promedio de años secos (cuatro de cada diez), años medios (cuatro de cada diez) y años húmedos (dos de cada diez) nos permite visualizar conceptualmente dichos excedentes (figura 6). 


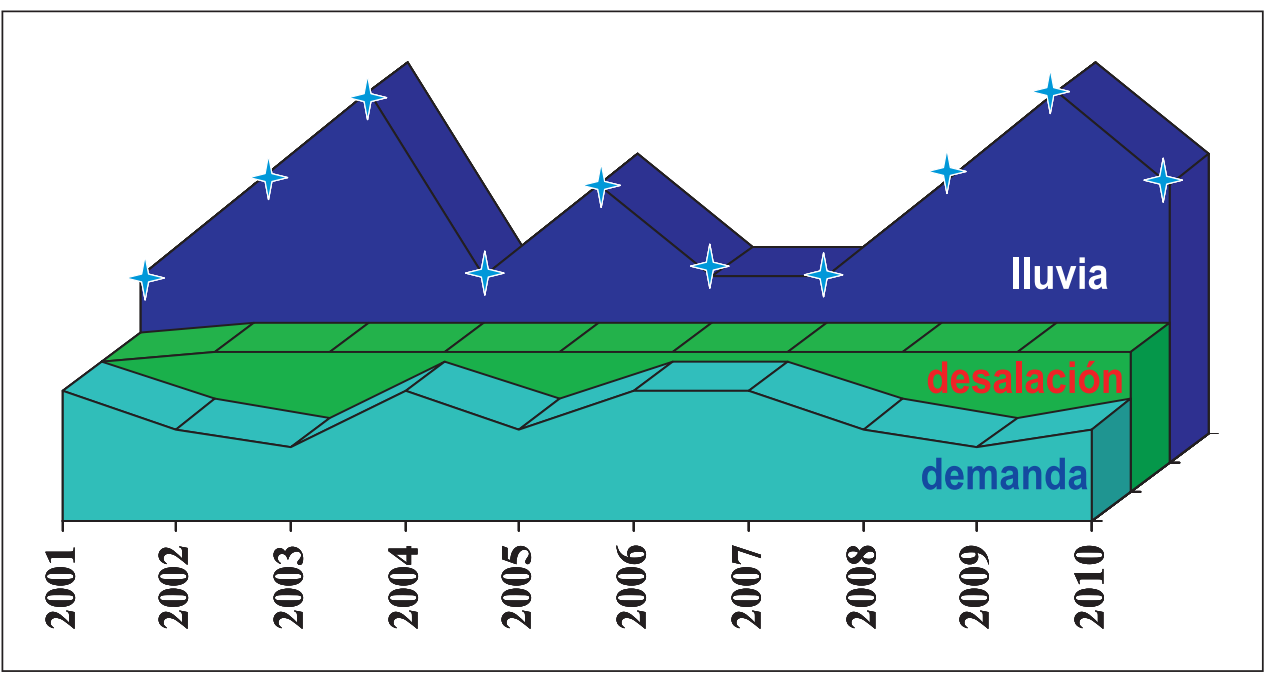

FIgURA 6: Simulación de los excedentes de producción de agua desalada en Baleares en un periodo climatológico promedio.

No obstante, el principal problema, aparte del consumo energético, para aplicar criterios de eficiencia en la producción de las desaladoras reside en ser consciente que, al contrario de lo que hasta ahora ocurre con la producción eléctrica, el agua puede ser almacenada. Ello conlleva que el dimensionado de dichas plantas no ha de hacerse en relación con las puntas de la demanda, sino que debe establecerse de acuerdo con las necesidades promedio.

\section{El almacenamiento en acuíferos}

Para una gestión eficiente de la producción continuada de las desaladoras, resta por resolver el problema de dónde almacenar el agua producida en la temporada de bajo consumo, para poder utilizarla en las puntas de mayor demanda. Podría pensarse en su acumulación en embalses superficiales, cuyos inconvenientes ya han sido comentados antes, sin embargo, los acuíferos resuelven dichos problemas y aportan una mayor capacidad. Tan sólo requieren un buen conocimiento de su geología que nos permita conocer su geometría, determinar su volumen y su funcionamiento.

En las islas Baleares se ha podido ensayar el almacenamiento en acuíferos cársticos. La existencia de un proyecto de trasvase de los caudales vertidos al mar desde una surgencia cárstica en la costa norte de Mallorca hacia Palma, puso de manifiesto la necesidad de encontrar un almacenamiento para los volúmenes trasvasados. De hecho, el proyecto, conocido como trasvase de sa Costera, no tenía sentido sin encontrar un sistema de almacenamiento hiperanual: los caudales más importantes aportados por la fuente se producen en los inviernos lluviosos, cuando las posibilidades de ser utilizados por el abastecimiento urbano o agrícola son inexistentes. Así pues, se modificó el proyecto y en él se introdujo un sistema para la infiltración de agua en el acuífero de s'Estremera, próximo al trazado de la conducción (véase la figura 10). Con el fin de comprobar el funcionamiento de dicho acuífero, durante el invierno del año 2002 se infiltraron casi $6 \mathrm{hm}^{3}$ de agua en s'Estremera 


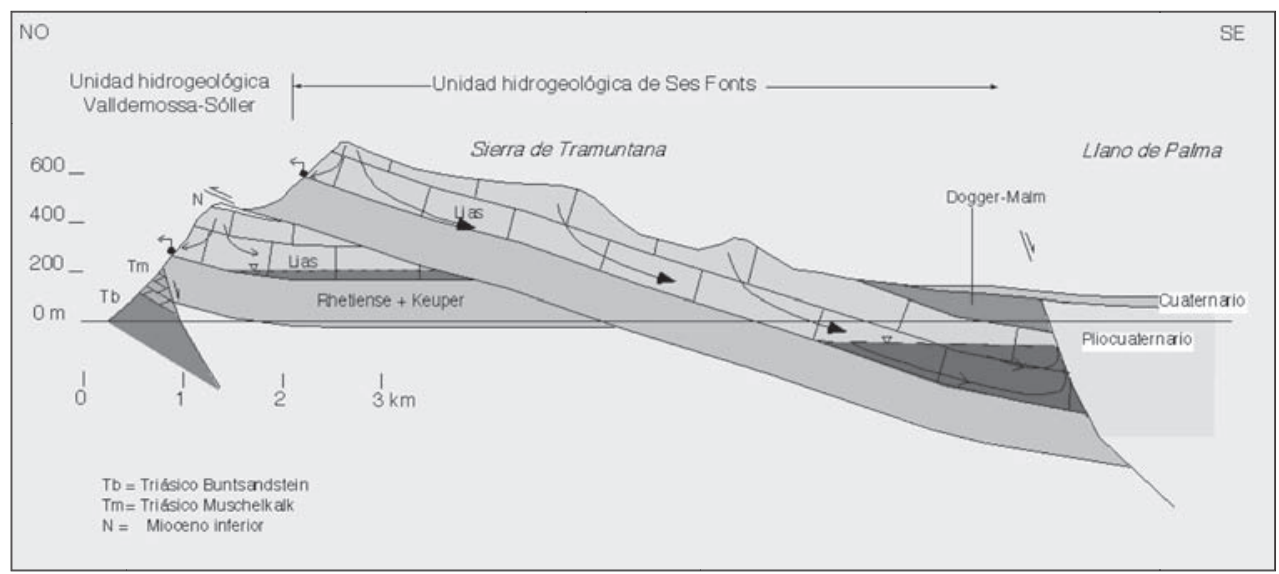

FIgURA 7: Corte representativo del acuífero de ses Fonts según GELABERT y SABAT (2003).

y se comprobó a través de la evolución de sus niveles piezométricos (figura 3) que su geometría respondía a los estudios previos y que su estanqueidad era real.

En la figura 7 se representa la geometría del acuífero de ses Fonts con niveles piezométricos bajos, tal y como se encontraría en un evento seco o después de un periodo de fuertes extracciones. Su disposición estructural es similar a las del acuífero de s'Estremera (fig. 3) o al de Crestatx (fig. 10) Cuando se producen fuertes lluvias los niveles piezométricos pueden recuperarse produciéndose en los pozos más bajos del acuífero ascensos por encima de la cota del sondeo.

\begin{tabular}{|c|c|c|c|c|c|c|c|c|c|c|c|c|c|c|c|}
\hline IDAM & 1.994 & 1.995 & 1.996 & 1.997 & 1.998 & 1.999 & 2.000 & 2.001 & 2.002 & 2.003 & 2.004 & 2.005 & 2.006 & 2007 & 2.008 \\
\hline Formentera $\left(2^{*} 1000+1^{*} 2000\right)$ & & & 0,3 & 0,7 & 0,7 & 0,7 & 0,7 & 0,7 & 1,0 & 1,4 & 1,4 & 1,4 & 1,4 & 1,4 & 1,4 \\
\hline$(33000)$ & 1,3 & 3,1 & 3,1 & 3,1 & 3,1 & 3,1 & 3,1 & 3,2 & 3,2 & 3,2 & 3,2 & 3,2 & 3,2 & 3,2 & 3,2 \\
\hline$\left(23^{*} 4000\right)$ & & & 1,2 & 2,8 & 2,8 & 2,8 & 2,8 & 2,8 & 2,8 & 2,8 & 2,9 & 4,3 & 4,3 & 4,3 & 4,3 \\
\hline Son Tugores (aigües salobres) & & 7,2 & 10,8 & 13,0 & 13,0 & 13,0 & 15,1 & 15,1 & 15,1 & 15,1 & 15,1 & 15,1 & 15,1 & 15,1 & 15,1 \\
\hline$\left(9^{4} 7200\right)$ & & & & & & 7,6 & 17,4 & 20,6 & 22,6 & 22,6 & 23,3 & 23,3 & 23,3 & 23,3 & 23,3 \\
\hline Modulars & & & & & & & 1,1 & 1,3 & 1,3 & 1,3 & 1,3 & 1,3 & 1,3 & 1,3 & 1,3 \\
\hline Camp de Mar $(2 * 2100+2+7000)$ & & & & & & & & & 0,9 & 0,9 & 0,9 & 0,9 & 0,9 & 0,9 & 5,0 \\
\hline Son Ferrer & & & & & & & 0,5 & 1,9 & 1,9 & 1,9 & 2,0 & 2,0 & 2,0 & 2,0 & 2,0 \\
\hline$(27000)$ & & & & & & & & & & & & & 5,0 & 5,0 & 5,0 \\
\hline Santa Eulalia & & & & & & & & & & & & & 5,0 & 5,0 & 5,0 \\
\hline Civtadella & & & & & & & & & & & & & & 5,0 & 5,0 \\
\hline Total & 1,3 & 10,3 & 15,4 & 19,5 & 19,5 & 27,1 & 40,6 & 45,6 & 48,9 & 49,2 & 50,1 & 51,6 & 61,7 & 66,7 & 70,8 \\
\hline
\end{tabular}

Figura 8: Capacidad de desalación instalada o prevista en las islas Baleares entre 1994 y 2008. 
La importancia de la infiltración de agua en el acuífero de s'Estremera resulta evidente en un archipiélago en el que predominan las litologías calcáreas y en el que la producción de agua desalada tiene un fuerte componente estacional. La primera planta desaladora en las islas Baleares se construye en Ibiza y empieza su producción en el año 1994, un año después, inicia su producción la planta de aguas salobres de son Tugores en Mallorca y en 1996 la planta desaladora de Formentera. La evolución del parque público de desaladoras (fig. 8) aumenta rápidamente y en una década pasamos de la primera planta de Ibiza a ocho instalaciones que alcanzan una capacidad de producción de $50 \mathrm{hm}^{3} /$ año. Las previsiones permiten afirmar que en torno al 2008 las Islas tendrán un parque de once plantas y una capacidad de producción de $70 \mathrm{hm}^{3} /$ año. Esto representa un $70 \%$ de la demanda urbana de las Baleares en la actualidad.

Otra cosa es la producción de dichas plantas. En la figura 9 puede observarse cómo después de un periodo inicial en el que se utiliza la práctica totalidad de la producción, la instalación de nuevas desaladoras y la ampliación de las existentes nos lleva a una situación de sobrecapacidad, en la que sólo se utiliza un $65 \%$ de la producción potencial. Esto sucede incluso coincidiendo con una de las mayores sequías registradas en las Baleares (véase la figura 1). Acabada la sequía, a finales del 2001, la sobrecapacidad se pone aún más de manifiesto y alcanza el doble de la producción. Finalmente cuando se disponga de todas las plantas desaladoras en producción su sobrecapacidad puede estimarse llegue al $230 \%$.

En esta situación, parece necesario responder a una pregunta: ¿Cuál es el sentido de disponer de una capacidad de producción que dobla el consumo previsible de agua desalada? La respuesta tradicional ha sido la de afrontar las puntas de demanda estivales. No obstante, hoy sabemos que dicha respuesta representa un despilfarro de recursos, pues, pudiendo almacenarse el agua producida a lo largo del año, el diseño razonable debiera de ajustarse a las necesidades promedio y no a las puntas. Sin embargo, dado que ya se dispone de

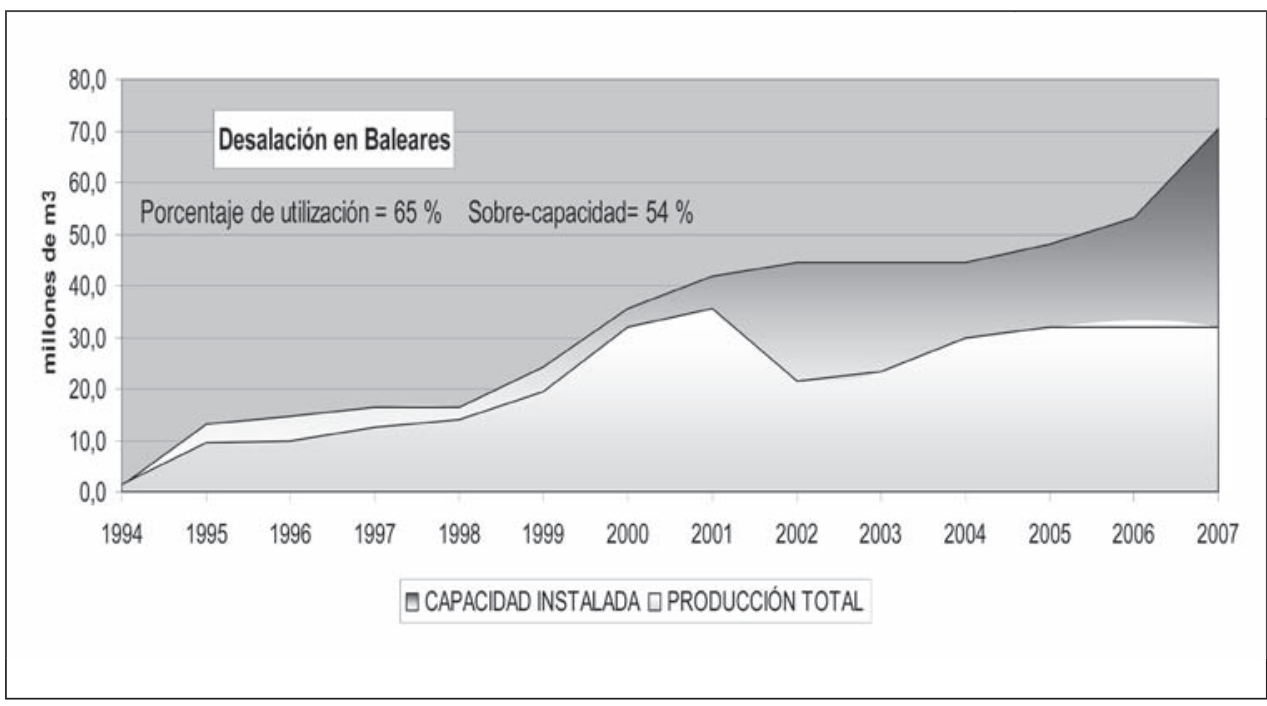

Figura 9: Capacidad y producción de las plantas desaladoras de las Baleares. Entre 1994 y 2004 los datos corresponden a valores reales suministrados por el Instituto Balear del Agua y la Energía (IBAEN) mientras que los datos entre el 2004 y 2007 son estimaciones de los autores. 
una capacidad de producción instalada de $50 \mathrm{hm} 3 / a n ̃ o$, caben otras respuestas. La primera representa utilizar la sobrecapacidad instalada para acumular una reserva estratégica de agua que, no sólo permitiría una seguridad en el suministro urbano, sino también posibilita abaratar los costes de producción por metro cúbico. La segunda, perfectamente compatible y complementaria de la anterior, se refiere a poder utilizar esta sobrecapacidad para recuperar nuestros ecosistemas acuáticos. Ello representa poder cumplir con la Directiva Marco del Agua y así, devolver a nuestros acuíferos aquellas reservas no renovables que a lo largo de muchos años de sobreexplotación les hemos ido ‘quitando'. Pero, el hecho de recuperar los ecosistemas acuáticos significa, además, la mejor garantía de calidad y cantidad en el suministro a la población, aparte de los valores intrínsecos, tanto sociales como económico y ecológicos que representan las fuentes y los arroyos recuperados.

\section{Integración de recursos}

El agua desalada no es el único recurso hídrico no convencional que debe integrarse en una gestión eficiente de nuestros recursos. Existen otras aguas que también merecen nuestra atención. Si queremos que la gestión de los recursos hídricos sea sostenible, es decir, que pueda ser usada y transmitida a las futuras generaciones sin mayor deterioro, hemos de gestionarlos de la manera más eficiente, eliminando el despilfarro y el mal uso. Para ello necesitamos integrar todos los recursos disponibles para poder utilizar en cada momento y en cada situación el más adecuado según las necesidades y la oportunidad.

\subsection{Recursos integrables}

Queda fuera de este artículo el comentar ampliamente cada uno de estos recursos alternativos o poco usados, no obstante sí conviene relacionarlos antes de ejemplificar su integración a través de la experiencia llevada a cabo en Baleares. Entre los principales recursos hídricos a integrar se encuentran los siguientes:

a) Gestión de la demanda

b) Recursos convencionales

i) Aguas superficiales

ii) Aguas subterráneas

c) Recursos no convencionales

i) Aguas pluviales

ii) Aguas nuevas (regeneradas)

iii) Aguas desaladas

El principal recurso hídrico no es tanto la disponibilidad de agua en sí, como la garantía de disponer de ella. Es en este sentido que conviene considerar la gestión de la demanda como uno de los principales 'recursos hídricos' de que disponemos. Baste decir que la mejora de la eficiencia en el uso del agua urbana está consiguiendo en muchas de las grandes ciudades disminuir el consumo total de agua a pesar de los incrementos de sus poblaciones. Justo es destacar las posibilidades de la gestión de la demanda para superar los episodios de sequía. Necesario resulta señalar los pocos esfuerzos que se realizan para su implementación y las dificultades que ello conlleva.

En cuanto a los recursos convencionales, sólo señalar que probablemente estamos sobrepasando los límites de su captación sostenible, no obstante queda mucho por hacer en lo que respecta a su transporte eficiente y a evitar su contaminación.

Los recursos no convencionales personifican el agua del futuro, tanto en los países desarrollados como en los menos desarrollados. Las aguas pluviales están ocupando un 
papel cada vez más importantes en muchas áreas del Globo: en el sur de Australia su recogida representa una de las soluciones más imaginativas para solventar diversos tipos de suministro en las zonas urbanas. Inevitable es recordar la cultura de los aljibes, presente todavía en nuestras áreas rurales y a la que un falso progreso parece estar volviendo la espalda. Las aguas nuevas o regeneradas constituyen un recurso inmenso, seguro, abundante y, especialmente útil en las puntas de demanda del verano, sobre todo en las agrícolas. Tan sólo una depuración poco rigurosa, lamentablemente bastante generalizada, ha impedido hasta ahora su desarrollo. Finalmente, las aguas desaladas ya forman parte del suministro en las zonas insulares, Canarias y más recientemente Baleares, y probablemente también lo hagan masivamente en el litoral mediterráneo peninsular. Preciso es señalar que su rápido desarrollo está relacionado con la facilidad de su instalación, si la comparamos con otras medidas, y a los avances tecnológicos en su funcionamiento. Esta misma facilidad y sus efectos paralizantes sobre otras medidas menos consumidoras de energía, reflejan sus inconvenientes principales.

\subsection{Condicionantes de la gestión integrada}

Para disponer de los recursos a integrar en una gestión conjunta, necesitamos resolver antes otros problemas que, aunque menores, pueden dificultar y hasta imposibilitar la gestión conjunta de los recursos disponibles. Requerimos determinar los siguientes puntos:

a) Infraestructuras de interconexión

b) Criterios operacionales

c) Regulador del sistema

d) Voluntad política

Las infraestructuras de interconexión son un requisito previo y obvio para proceder a una gestión conjunta de los recursos hídricos. No obstante, muchas de las infraestructuras ya existentes pueden orientarse, con relativa facilidad, a las necesidades de interconexión y aún con mayor facilidad, pueden diseñarse los nuevos proyectos para que contemplen dicha posibilidad.

Establecer criterios operacionales claros y consensuados resulta imprescindible para poder gestionar de forma integrada los recursos hídricos. A pesar de su carácter elemental, conseguir acordar dichos criterios no resulta fácil y más aún cuando en la gestión integrada intervienen entidades diversas, con intereses distintos y muchas veces de colores políticos distintos. Un aspecto positivo a señalar es la potencialidad de intercambios virtuales de agua entre los diversos elementos que componen el sistema; de hecho, más allá de pequeñas diferencias en la calidad, el metro cúbico de agua puede considerarse equivalente tanto si procede de aguas superficiales, subterráneas o desaladas.

A pesar de contar con los criterios operacionales más transparentes posibles, el acuerdo sobre un regulador del sistema integrado es indispensable para su funcionamiento. Siempre pueden producirse coyunturas temporales o espaciales que obliguen a rápidas decisiones que, por su propia naturaleza, no puedan ser sometidas a un proceso de discusión participativa. Entonces es cuando la existencia de un regulador mantiene el sistema operativo.

Finalmente, y no por más obvio, conviene citar como último requisito la voluntad política. Éste es un asunto que puede dar al traste con meses de negociaciones y acuerdos bien trabajados a nivel técnico, que luego por intereses más o menos racionales no pueden ponerse en práctica. 


\section{Una gestión integrada en baleares}

La demanda de agua en las islas Baleares puede cifrarse en poco más de $200 \mathrm{Hm}^{3} / a n ̃ o n$, que se reparten casi por igual entre el uso agrícola y el urbano. El crecimiento del consumo urbano - turístico en buena medida - se ha producido, primero, mediante la sobreexplotación de los acuíferos de las Islas y en los últimos años por el agua desalada. De hecho, el descenso de los niveles piezométricos y la salinización de los acuíferos costeros sería mucho mayor si no fuera por el descenso, en paralelo, del consumo agrícola (Plan Hidrológico de las Islas Baleares, 2002).

La demanda agrícola se satisface mediante aguas subterráneas (un $80 \%$ ) y aguas nuevas (un 20\%), mientras que la demanda urbana utiliza aguas superficiales (menos del 10\%), aguas subterráneas (un $70 \%$ aprox.) y aguas desaladas (un $20 \%$ aprox.). Varios proyectos se están desarrollando actualmente para utilizar aguas regeneradas en limpieza de calles y riego de jardines en Palma y Calvià. Una depuración todavía más rigurosa y sobre todo manteniendo constante su calidad, permitiría disponer de un recurso abundante, especialmente en las épocas más secas, apto para numerosos usos y con las cualidades previamente establecidas.

El principal problema que plantea la gestión del agua en las islas Baleares no es tanto la disponibilidad del recurso, cuanto su irregularidad. Es cierto que el consumo total ha ido aumentando al crecer la población, pero ya hemos comentado que el declive agrícola ha liberado numerosos recursos que compensan, en buena parte, el consumo urbano. En cambio, la alternancia de periodos secos y húmedos sí representa un reto para la gestión insular.

\subsection{La Arteria Transversal}

Más allá de establecer políticas destinadas a la disminución del consumo, reducción de fugas, etc., cuyo déficit es todavía ingente, la integración de los distintos tipos de recursos hídricos es la respuesta más eficiente a la alternancia climática. Para ello, y a modo de ilustración de cómo gestionar de forma integrada los recursos hídricos insulares, se describe a continuación la Arteria Transversal (figura 10) que con algunas modificaciones, continua construyéndose en Mallorca desde hace más de una década.

La Arteria Transversal de Mallorca interconecta los principales recursos hídricos de la Isla. Se sitúa al pie de la Sierra de Tramontana y transcurre desde Pollença (al NE de la cordillera) hasta Andratx (al SW). Aprovecha infraestructuras existentes y otras de reciente construcción y conecta las plantas desaladoras instaladas hasta el momento en Mallorca. Dispone de depósitos de regulación y de los mas importantes pozos de extracción y de infiltración de la Isla.

Por orden cronológico, integra dos pequeños embalses (Cuber y el Gorg Blau) situados en las partes más altas de la Sierra de Tramontana, próximos al Puig Major, junto con su conducción hasta Palma (tramo 1 de la figura 10). También integra la conducción Llubi - Palma (tramo 2 de la figura 10), también conocida como trasvase de sa Marineta, que permite de forma reversible enviar o traer caudales desde el acuífero principal de Mallorca (Unidad Inca-sa Pobla) hacia Palma o viceversa. Comprende la llamada Arteria de Ponent (tramo 3 de la figura 10), conducción también reversible entre la desaladora de la Badia de Palma y el poniente de Mallorca (Calvià y Andratx). La Arteria Transversal se completa con las conducciones del llamado trasvase de sa Costera (tramo 4 de la figura 10), proyecto en construcción, que conduce las aguas del manantial de sa Costera, en la costa NW de Mallorca, hasta Sóller, primero, hasta el acuífero de sa Extremera después, y finalmente 


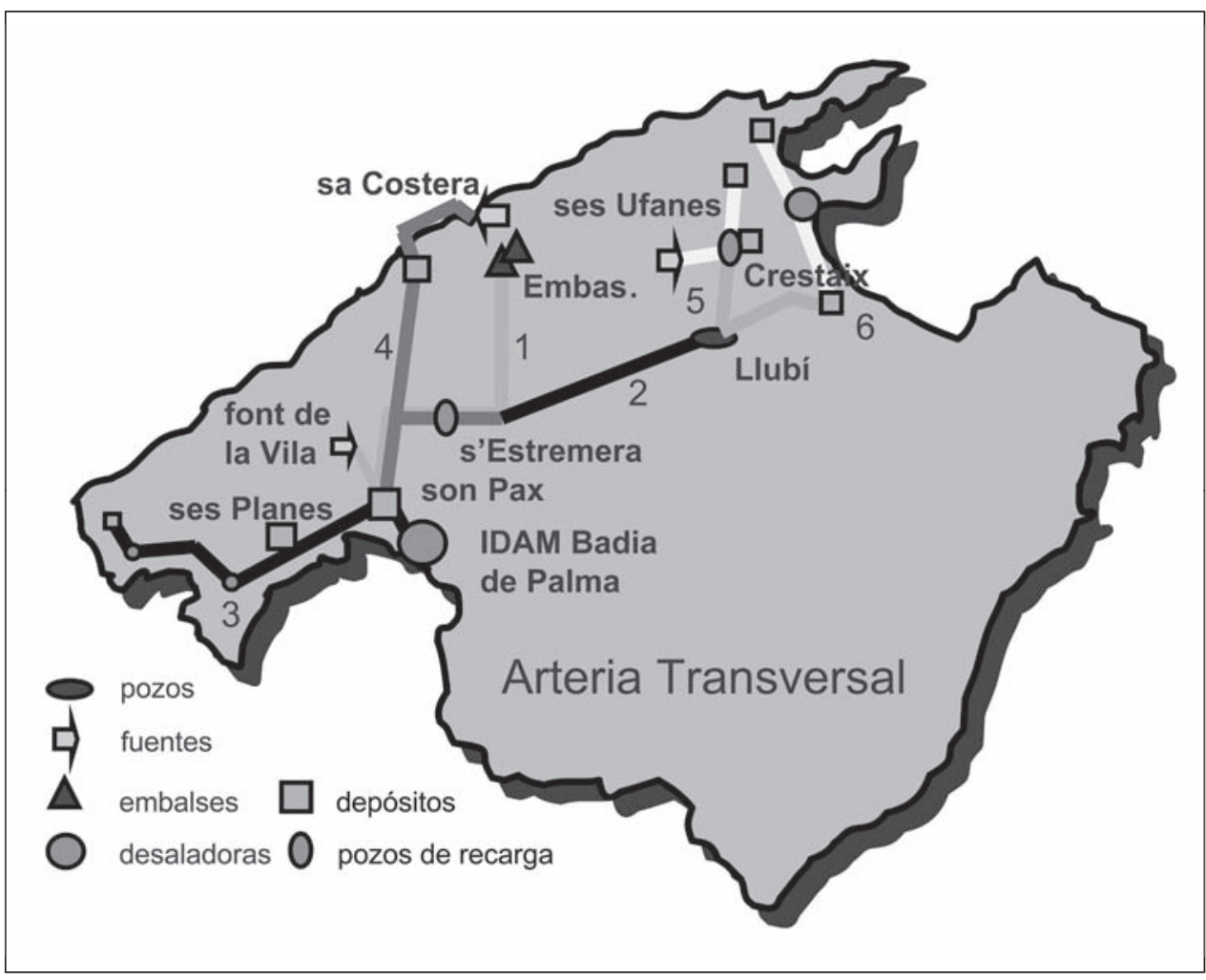

Figura 10: Esquema simplificado del diseño de la Arteria Transversal de Mallorca. La línea azul corresponde a tramos diseñados para el suministro de Palma. La línea negra representa tramos ya construidos. La línea roja corresponde al transvase de sa Costera, actualmente en construcción. Los tramos amarillos y anaranjados del Norte de la Arteria han sido recientemente modificados en función de una nueva desaladora a construir en Alcudia ( $\mathrm{N}$ de Mallorca).

hasta Palma. El tramo Norte de la Arteria Transversal (tramo 5 de la figura 10) se proyecto para llevar agua hasta y desde Llubí al acuífero de Crestatx y desde allí, hasta Alcudia y Pollença. Sin embargo, durante su ejecución fue modificado sustancialmente, variando su trazado y sus fines, para hacerlo coincidir con una nueva desaladora a instalar en Alcudia (tramo 6 de la figura 10).

Todo este conjunto permite disponer de agua de distintas procedencias para abastecer al $70 \%$ de la población de Mallorca y por tanto, lograrlo con la mayor eficiencia. Pero permite además, recuperar los acuíferos y las fuentes de buena parte de la Isla, ya que al ganar en eficiencia disminuimos la sobreexplotación y recuperamos paulatinamente los recursos disponibles. Para conseguirlo necesitamos, como ya se dijo anteriormente, acuerdos sustanciales en los objetivos y en los procedimientos. En cuanto a los objetivos resulta fácil ponerse de acuerdo, siempre y cuando no se produzcan perjuicios económicos en los diversos intereses en juego. En el caso que comentamos, el coste global de una mayor producción de agua desalada, se compensaba con un menor coste por metro cúbico producido y permitía, además, que nuevos municipios participaran de esa mayor producción. Más aún, la llegada 
de nuevos caudales procedentes del trasvase de sa Costera, que hasta ahora se vertían al mar, representa un plus de abaratamiento extra que debiera de utilizarse para garantizar la compra de la totalidad del agua desalada producida.

En definitiva, las compensaciones propias de la gestión integrada permiten obtener mayores recursos con los que compensar la sobreexplotación histórica de los acuíferos de buena parte de Mallorca, sin tener que penalizar extraordinariamente el precio del agua. Necesario es recordar que, aún con grandes desigualdades entre diversas zonas de las Islas, el precio del agua en Baleares es uno de los más altos de España.

Los acuerdos procedimentales son también imprescindibles. El principal de ellos requiere establecer las prioridades en el uso de los diversos recursos disponibles en cada momento. De forma secundaria, han de definirse como y cuando han de hacerse los trasvases, reales o virtuales, la contabilidad del agua recibida, entregada y/o almacenada, los actores de cada operación, etc. En cuanto a las prioridades generales, en el caso de Mallorca, se propusieron las siguientes:

1. Gestión de la Demanda (fundamentalmente gestión en baja)

2. Recursos No Convencionales (aguas desaladas y regeneradas)

3. Aguas superficiales

4. Aguas subterráneas

Con esta jerarquía se alcanza una recuperación progresiva de los acuíferos que, a medio plazo, llevará a la recuperación de fuentes y cursos superficiales efímeros y a un plazo ya mayor a la recuperación de algunos cauces permanentes. Siguiendo estos criterios se

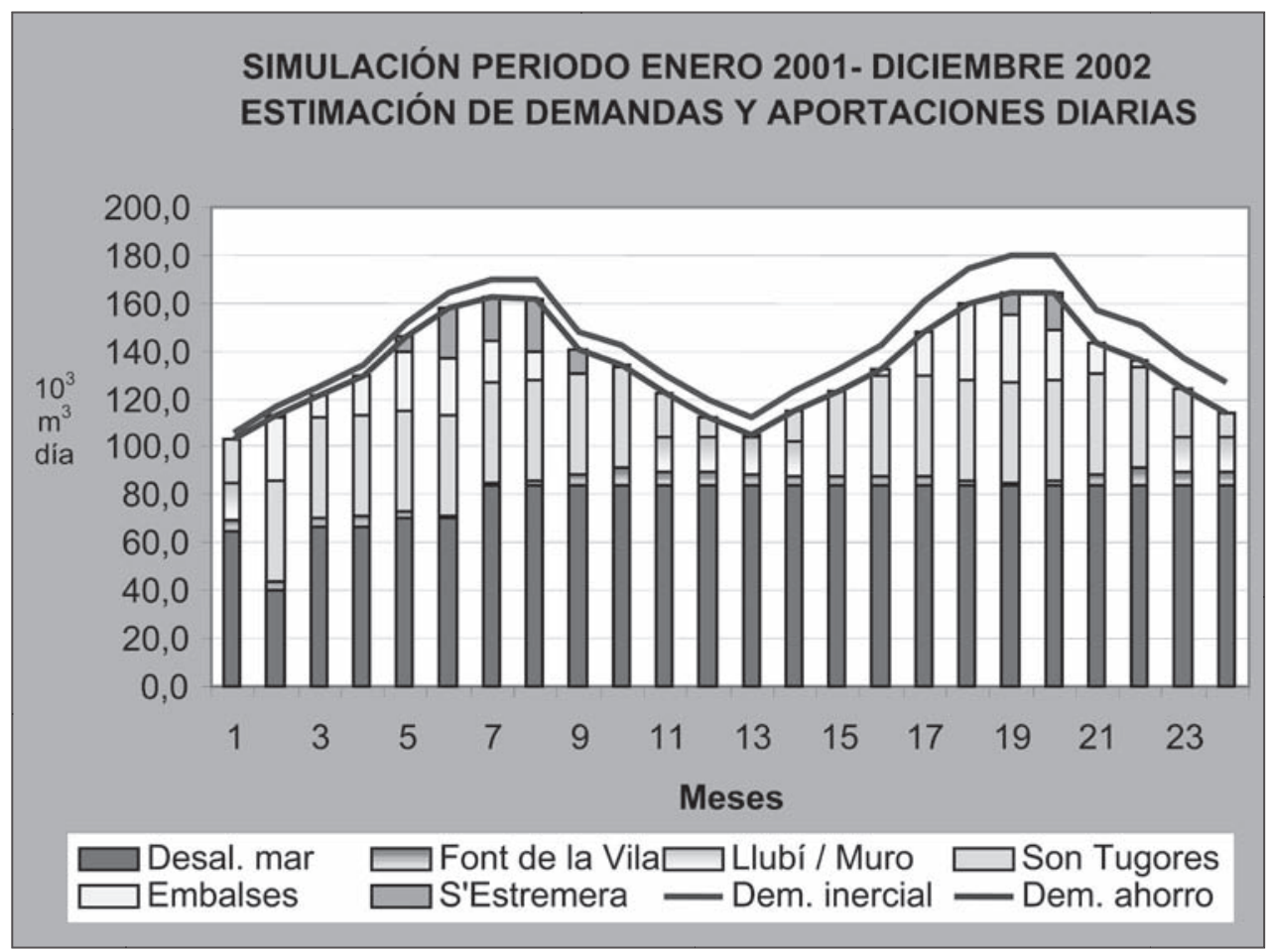

FIGURA 11: Simulación del abastecimiento a Palma durante los años 2001 y 2002 aplicando criterios de gestión integrada. 
procedió a una simulación del suministro urbano de Palma a lo largo de dos años (figura 11) y para precipitaciones promedio.

En primer lugar, sobre la curva de demanda inercial se ha previsto una disminución limitada, pero progresiva, correspondiente a la gestión de la demanda. Estas acciones corresponden a los ayuntamientos per se o a través de sus concesionarios. Sus posibilidades son en nuestro país, todavía inmensas y justo es reconocer que las mejoras tecnológicas en las nuevas viviendas están ya reduciendo pérdidas y desarrollando consumos más eficientes.

A partir de la curva que ha resultado de la aplicación de una eventual gestión de la demanda sobre la demanda inercial prevista, obtenemos la demanda con ahorro (figura 11). Ahora debemos rellenar cada demanda mensual con los recursos existentes: en una primera instancia colocamos la producción de agua desalada generada a partir de agua de mar. A continuación la generada a partir de aguas salobres (IDAM de son Tugores) y seguidamente las aguas superficiales y las subterráneas. Puntualmente situamos aguas procedentes del acuífero del Norte (Llubí/Muro) que en dichos años no podían utilizarse en la zona de procedencia por falta de infraestructuras y las aguas procedentes de manantiales próximos a la ciudad de Palma. El resultado, en sólo dos años, ya refleja la disminución de las extracciones en el acuífero de s'Estremera y por tanto, el inicio de su recuperación en un escenario de precipitaciones normales.

\section{Conclusiones}

El ejemplo de gestión integrada del agua en las islas Baleares pone de manifiesto sus virtudes para favorecer un uso eficiente de nuestros recursos. La eficiencia es una condición necesaria para aumentar la sostenibilidad global. Además posibilita la utilización del mejor recurso disponible en cada momento, con ello se consigue, a medio plazo, recuperar los ecosistemas acuáticos y así, obtener las garantías de calidad y cantidad, objetivos de la Directiva Marco del Agua.

De especial interés resulta la experiencia de infiltración en acuíferos. A partir de estas posibilidades de almacenamiento, se posibilita una gestión hiperanual de los recursos hídricos, absolutamente necesaria en climas mediterráneos caracterizados por la alternancia entre periodos húmedos y secos.

Resulta decisivo para tener éxito en tal gestión, dotarse de las infraestructuras de interconexión necesarias, acordar los criterios operacionales, consensuar el regulador del sistema y la voluntad política. La importancia de esta última es indudable y para ponerlo de manifiesto, baste decir que el cambio político último acontecido en las Baleares ha modificado las prioridades acordadas por el Gobierno anterior y ha retornado a políticas sectoriales que, a modo de compartimentos estancos, favorecen de nuevo las soluciones tecnocráticas. El manejo integrado se mantiene como proyecto, pero sus objetivos olvidan, a nuestro criterio, la gestión hiperanual y el marco ecointegral, absolutamente necesarios para el cumplimiento de los objetivos de la Directiva Marco.

\section{Agradecimientos}

La mayoría de los datos que se ofrecen en este artículo, muchos de ellos únicamente recogidos en informes internos, han sido obtenidos por técnicos y personal de la Direcció General de Recursos Hídricos del Govern de les Illes Balears. A todos ellos nuestro agradecimiento más sincero. 


\section{Bibliografía}

GELABERT, B. y SABAT, F., (2002): «Relaciones entre la hidrología subterránea y la estructura geológica de la sierra de Tramontana de Mallorca (Islas Baleares)», Geogaceta, 31, 107-110.

GLEICK, P. H., (1996): «Water resources», en Encyclopedia of Climate and Weather, ed. by S. H. Schneider, Oxford University Press, New York, vol. 2, pp. 817-823.

INSTITUTO NACIONAL DE METEOROLOGÍA DE ESPAÑA - INME Centro Territorial en Palma de Mallorca (2005): Seguimiento de la sequía.

PLAN HIDROLÓGICO DE LAS ISLAS BALEARES (2002), Direcció general de Recursos Hídrics, Conselleria de Medi Ambient. Govern de les Illes Balears.

UNITED STATES GEOLOGICAL SURVEY - USGS, (2004): The water cycle. http://ga.water.usgs. gov/edu/watercyclesummary.html 